\title{
Resiliencia en adolescentes con ideación suicida
}

\section{Resilience in adolescents with suicidal ideation}

Resiliencia en adolescentes

Neycer Bladimir Aldaz García ${ }^{(1)}$

Gustavo Rafael Escobar Delgado ${ }^{(2)}$

(1) Universidad Técnica de Manabí. Portoviejo. Ecuador. email: bladimiraldaz7@gmail.com

(2) Universidad Técnica de Manabí. Portoviejo. Ecuador. email: gustavoescobar_2006@hotmail.com

Contacto: bladimiraldaz7@gmail.com

Recibido: 12-5-2020

Aprobado: 4-7-2020

\section{Resumen}

El objetivo del estudio fue identificar los niveles de resiliencia que presentan los adolescentes con ideación suicida, de 15 a 16 años de edad de la unidad educativa fiscal "José Joaquín de Olmedo" de la ciudad de Portoviejo, Ecuador. El estudio es descriptivo, con metodología analítica-sintética, se utilizaron dos instrumentos con alto nivel de fiabilidad: la escala de ideación suicida de Beck y el test SV- RES de Saavedra y Villalta. Los principales destacan que el 30\% de adolescentes evaluados, presenta ideación suicida, mientras que, el $60 \%$ tiene resiliencia promedio lo que significa una adecuada capacidad de afrontamiento a las adversidades; y el 36.66\% de adolescentes tiene un nivel bajo de resiliencia; con escasa capacidad de afrontamiento. Se distinguen las diferentes etapas del suicidio y se evalúan los diferentes factores presentes en la ideación suicida, teniendo en cuenta sus variables. Se categorizan los niveles resilientes que presentan los estudiantes con ideas suicidas, determinándose el estado de riesgo en que se encuentran. En este contexto, el estudio fortalece las vertientes de la salud mental y al ámbito psicosocial, potenciando la necesidad de una observación más atenta y amplias a las ideas que presentan los adolescentes; además, se identifica los factores predisponentes, directos e indirectos, que conllevan a la ideación suicida. 
Palabras clave: Suicidio, ideación suicida, adolescentes

\begin{abstract}
The objective of the study was to identify the levels of resilience presented by adolescents with suicidal ideation, 15 to 16 years of age from the fiscal educational unit "José Joaquín de Olmedo" in the city of Portoviejo, Ecuador. The study is descriptive, with analytical-synthetic methodology, two instruments with a high level of reliability were used: the Beck scale of suicidal ideation and the SV-RES test of Saavedra and Villalta. The main ones highlight that $30 \%$ of evaluated adolescents present suicidal ideation, while $60 \%$ have average resilience, which means an adequate capacity to cope with adversity; and $36.66 \%$ of adolescents have a low level of resilience; with little coping capacity. The different stages of suicide are distinguished and the different factors present in suicidal ideation are evaluated, taking into account its variables. The resilient levels that students with suicidal ideas present are categorized, determining the state of risk in which they are. In this context, the study strengthens the aspects of mental health and the psychosocial field, strengthening the need for a more careful and comprehensive observation of the ideas presented by adolescents; In addition, direct and indirect predisposing factors that lead to suicidal ideation are identified.
\end{abstract}

Keywords: Suicide, ideation suicide, adolescents.

\title{
Introducción
}

El suicidio es una de las causas de muerte a nivel mundial, que constituye un acto deliberado de quitarse la vida; de allí, la cardinal importancia de estudiar los procesos que conllevan su concreción. Existen vestigios explicativos en torno al suicidio; donde varias son las teorías que tratan de explicar su origen, desarrollo y consumación; entre ellas las psicológicas, el psicoanálisis con Freud, quien destaca una orientación primaria hacia el objeto amado y las psicosociales, como por ejemplo el enfoque de Emile Durkheim, quien sostiene que el suicidio es producto de la influencia recíproca entre el individuo y la sociedad. En este rápido caminar histórico-epistemológico, la ideación es el primer momento del suicidio, comenzando con ideas autodestructivas o ideas de muerte (Quinlan, Sanhueza, Espinosa, Escamilla, Maddaleno, 2014).

El interés sobre las ideas suicidas en adolescentes, se fundamenta en la consideración de lo importante que es el conocimiento y la indagación de las causas, efectos y variables que se ponen a relieve ante tal 
fenómeno. Estas ideas se caracterizan por la aparición de pensamientos cuyo contenido se basa en terminar con su propia vida; pensamientos que varían desde la falta de valoración de la vida, deseo de terminar con su existencia, fantasías en torno a la muerte y la planificación del acto suicida propiamente dicho (De la Torre Marti, 2013).

La ideación suicida como etapa inicial del suicidio es un fenómeno multifactorial, complejo e interrelacionado en donde intervienen tanto factores psicológicos, sociales, culturales y biológicos. En este sentido, la identificación de estos factores en relación con el comportamiento suicida, es esencial para la prevención del suicidio, formando parte sustancial del sistema de la sanidad mental en grupos sociales prima el desarrollo armónico del psiquismo humano.

Dentro de las variables psicológicas asociadas con la ideación suicida, se encuentra: depresión, autoestima, consumo de drogas, desórdenes alimenticios, otros tipos de violencia, factores sociales, contexto familiar y contexto escolar.

Pereira y Cardoso (2015), hacen referencia algunos factores asociados a la ideación suicida, demuestran que son eventos multifactoriales o multidimensionales. Se han encontrado aspectos más subjetivos tales como: la falta de esperanza, la impulsividad, la agresividad, la percepción del aspecto corporal, las dificultades de comunicación y la sensación de pertenencia social, posiblemente son los que desencadenan el proceso de la ideación suicida.

Se plantearon otros factores como: las variables demográficas y socioeconómicas, orientación sexual, práctica religiosa, antecedentes del comportamiento suicida en la familia y amigos, consumo de sustancias psicotrópicas y síntomas depresivos.

A nivel nacional en el año 2017 hubo un incremento de suicidios existiendo un sub-registro del 16\% de intentos de suicidio. Al tomar en cuenta esta estadística, se evalúa el riesgo de suicidio en adolescentes de la ciudad de Portoviejo-Manabí.

Más allá del vector filosófico-epistemológico en que se ubiquen los letrados de la salud mental, lo cierto es que, la adolescencia es una etapa de cambios acompañados de variaciones físicas y emocionales, desarrollando también nuevas capacidades. Esta etapa es una de las más vulnerables para la aparición de conductas de riesgo. No solo es una etapa de vulnerabilidad sino también de oportunidad, porque aprendiendo a gestionar las emociones y a desarrollar las capacidades resilientes y 
de enfrentamiento, se puede contribuir al enfrentamiento apropiado de estímulos negativos, riesgos y adversidades de todo tipo.

Estos niveles influyen la capacidad resiliente en los adolescentes con ideación suicida. Identificando a los adolescentes con ideación suicida para luego determinar sus capacidades de afrontamiento a estas dificultades o a la identificación de sus capacidades resilientes. Los adolescentes son portadores de una carga emocional y cognitiva que los predispone para vivir las más versátiles aventuras en el trayecto hacia la conquista de sus sueños.

El presente trabajo tiene por objetivo identificar los niveles de resiliencia que presentan los adolescentes con ideación suicida, de 15 a 16 años de edad de la unidad educativa fiscal "José Joaquín de Olmedo" de la ciudad de Portoviejo, Ecuador.

\section{Materiales y métodos}

La población fue de 100 estudiantes de la unidad educativa fiscal "José Joaquín de Olmedo" de la ciudad de Portoviejo, los que fueron de manera intencional. El estudio es realizado con un carácter descriptivo, utilizando los métodos de inducción-deducción, análisis-síntesis. La investigación tiene un enfoque mixto (cuanti-cualitativo), realizado durante el primer trimestre de 2020, comenzando con la indagación de la ideación suicida de los adolescentes a través de la aplicación de la escala propuesta por Beck, que, metodológicamente, orienta la identificación de las ideas autolíticas que presentan los adolescentes sujetos de investigación.

Una vez identificados los adolescentes con persistentes ideas suicidas, mediante el test SV - RES planteado por Saavedra y Villalta, se evalúa el nivel de resiliencia que tienen los adolescentes, pudiendo ser alto, medio y bajo. Asimismo, se aplicó una entrevista en profundidad con la finalidad de obtener un plus indagatorio relevante que fortaleciera la información proporcionada por la escala y el test.

Se emplearon materiales tecnológicos como: calculadora, laptop o computadora, el programa de Word, el programa Excel para la tabulación de resultados. 


\section{Resultados}

El estudio fue realizado a 100 adolescentes de la unidad educativa fiscal "José Joaquín Olmedo", de la ciudad de Portoviejo, Manabí, en edades de 15 a 16 años. Los resultados logrados con la aplicación de la escala de ideación suicida de Beck, expresan que el 30\% de los estudiantes evaluados presentan ideación suicida, conviene reiterar que, estos resultados son producto del análisis de la mencionada escala, tabla 1.

Para el presente estudio se toma en consideración solo los adolescentes con ideación suicida, formando estos el 30\% de la población.

Posteriormente, mediante el test SV- RES de Saavedra y Villalta, se evaluaron los niveles de resiliencia, obteniendo como resultados que el 60\% de los adolescentes demuestran una resiliencia promedio, lo que significa que este porcentaje de estudiantes ofrece capacidad de afrontamiento a las adversidades; mientras que, el 36.66\% de adolescentes investigados exhibe un bajo nivel de resiliencia, lo cual los torna más susceptibles a llevar a cabo sus ideas suicidas, por cuanto en ellos no se haya convenientemente desarrollada la capacidad de afrontamiento.

\begin{tabular}{|l|l|}
\hline Con ideación suicida & Sin ideación suicida \\
\hline 30 adolescentes & 60 adolescentes \\
\hline
\end{tabular}

Tabla 1. Resultados de la escala de ideación suicida de Beck

Por último, el 3.33\% muestra un nivel alto de resiliencia. Lo que quiere decir que estos adolescentes poseen una excelente capacidad de afrontamiento frente a las adversidades que plantea el dolor emocional y en este caso frente a la ideación suicida; incluso pueden salir fortalecidos de tales contratiempos, tabla 2.

\begin{tabular}{|l|l|}
\hline Tipos de niveles de resiliencia & $\begin{array}{l}\text { Niveles de resiliencia en los } \\
\text { adolescentes con ideación suicida }\end{array}$ \\
\hline Alto & $3.33 \%$ \\
Promedio & $60 \%$ \\
Bajo & $36.66 \%$ \\
\hline
\end{tabular}

Tabla 2. Resultados de escala SV-RES de Saavedra y Villalta 
En los adolescentes con ideación suicida predomina un tipo de nivel promedio, con un resultado del $60 \%$. Teniendo en cuenta este resultado se evidencia que estos adolescentes cuentan con las capacidades para evitar llegar a realizar un acto o consumar el suicidio.

En base a la entrevista en profundidad se pudo evidenciar que ciertos adolescentes tenían respuesta como "ya para que seguir viviendo, mejor sería morir y así se termina todo". Mientras que otros mencionaban que "no deseaban morir y que la vida es maravillosa". Una información relevante, que de primera mano se obtuvo, es referente a los complejos fenómenos que negativamente padecen e inciden en estos adolescentes, como son: los contratiempos interfamiliares, las dificultades económicas y, en algunos casos, ciertos rasgos depresivos.

\section{Discusión}

Es pertinente destacar que la investigación realizada acerca de la ideación suicida en adolescentes, se diferencia de otros estudios porque está enfocada sólo en la primera etapa que justamente se denomina idea suicida, tal como lo plantea Miranda (2009); luego, estas ideas han sido relacionadas con la capacidad de afrontamiento o la resiliencia del ser humano. Mientras que otras indagaciones se centran más en los planes, conductas y en el suicidio consumado, este estudio se enfoca en la antesala de la autoeliminación; de allí, la importancia que reviste identificar, las ideas constantes de autolesión o de llevar a cabo, mediante un plan, el suicidio.

Según la OMS (2014), cada 30 segundos se suicida una persona en el mundo y cada día hay en promedio casi 3000 que ponen fin a su vida, y al menos 20 intentan suicidarse por cada una que lo consigue. Sobre el suicidio existen diferentes aspectos evaluados en los últimos años, tales como los comportamientos asociados, intentos previos, planeación de los mismos e ideación suicida; así mismo, los factores de riesgo sociodemográficos como edad, estado civil, escolaridad, historia laboral y el central para este análisis, el género.

Se concuerda con la investigación realizada por Rodríguez, Medina y Cardona (2013), se evidencia que "cada 40 segundos hay un suicidio en alguna parte del planeta y que cada tres segundos una persona intenta quitarse la vida", independientemente de la edad e ideación suicida, apoyo social y resiliencia en jóvenes.

Por otra parte, Freud (1920) citado por Barrionuevo, (2009) hace referencia: “'La relación que existiría entre el suicidio y un estado de duelo en la dimensión de la melancolía, la denigración de sí mismo, inequívocamente gozoso, importaría la satisfacción de tendencias sádicas y de odio que recaen primariamente sobre el objeto y que experimentarían una vuelta hacia la propia persona y dicho goce 
estaría sustentado sobre la base de una identificación narcisista. Para el enigma del suicidio Freud destaca que la energía psíquica para matarse derivaría del deseo de matar a alguien con quien se ha identificado, volviendo hacia sí dicho deseo de muerte',

Así mismo, la teoría cognitiva del modelo y comportamiento suicida de Beck, se basa en explicar las psicopatologías como la depresión y la ansiedad, pero intentando integrar nuevos elementos o constructos que ayuden a distinguir entre un individuo con riesgo suicida de otro que no lo tenga. En este sentido la teoría cognitiva de Beck, dice que los pensamientos y la manera de interpretar los eventos de la vida, tienen un rol causal en las respuestas emocionales y conductuales a tales eventos (Beck, 2008).

Según Linehan (1993) citado por Ortega (2018) afirma: "Todo aquel que se suicida busca la muerte como una solución única y definitiva para detener el sufrimiento”. De acuerdo con este autor, en la mayoría de los casos la cognición es la principal causa de los suicidios, pues en ella se lleva a cabo la actividad que regula el comportamiento. Sin embargo, la cognición no regula de forma absoluta en todos los contextos. Los estímulos ambientales también regulan, o incluso pueden llegar a provocar cogniciones y emociones problemáticas. Entonces, con base en lo anterior, afirma que la cognición es la mediadora entre lo ambiental y el comportamiento. Por otro lado, esta teoría sostiene que algunas personas suicidas son producto de cierta vulnerabilidad biológica que consiste en la falta de regulación de las emociones desde la etapa infantil y que puede ser detonada sin estar conscientes.

Durkheim (1960) citado por Rodríguez (2016) definió como "Todo caso de muerte que resulta directa o indirectamente de un acto positivo o negativo realizado por la víctima y que, según ella sabía, debía producir este resultado".

Durkheim (1960) hizo una distinción entre 3 tipos de suicidio:

1. El suicidio anómico: Es aquel en el que influyen tanto las crisis económicas como las crisis de la viudez o de vinculación.

2. Suicidio egoísta: Aquel que es proporcional al grado de integración de los grupos de los que el individuo es parte. La intensidad de los vínculos religiosos, familiares y políticos frena la tendencia a la autodestrucción suicida de quienes puedan sentirse tentados por ella. En definitiva, la integración actúa como freno a las fuerzas sociales suicidogénicas.

3. Suicidio altruista: Este tipo de suicidio sucede por una excesiva integración del sujeto con la sociedad a la que pertenece, a tal punto que la personalidad individual no cuenta. El individuo se 
somete con abnegación a las leyes y costumbres de su sociedad, de tal modo que se suicida porque piensa que ese acto es un deber.

Este tipo de suicidios se da según el autor, cuando la importancia del "yo" es muy baja. Es la versión opuesta al suicidio egoísta y existen 3 tipos:

3.1 Suicidio Altruista obligatorio: El que está condicionado por la sociedad, una sociedad que exige el suicidio. Por ejemplo, en sociedades asiáticas (normalmente relacionado con temas sobre el honor); o antiguamente, cuando en los pueblos celtas, y parece que también recientemente en nuestro entorno, los ancianos se quitaban la vida para no representar una carga para la sociedad en la que vivían. En este tipo de casos, la sociedad podría, en ocasiones, despreciar a la persona, si no lleva a cabo el suicidio.

3.2 Suicidio Altruista facultativo: La situación sería parecida a la anterior, con la diferencia de que la sociedad no demanda el suicidio del individuo. Por ejemplo, una viuda que se quita la vida, pero no por problemas psicológicos, sino porque siente que ya ha cumplido con su cometido en la vida.

3.3 Suicidio Altruista agudo: Cuando la persona se quita la vida por placer. Es común que se dé este caso en relación con temas religiosos. Por ejemplo, alguien que se suicida para alcanzar una supuesta gloria divina.

Spiller (2005) citado por Rodriguez (2016) considera el suicidio como: "el acto consumado, intencional de finalizar con la propia vida. Incluye todas las muertes que son resultado directo o indirecto de comportamientos ejecutados por la propia víctima, quien es consciente de la meta que desea lograr".

En este estudio, entre los adolescentes, fue evidenciada la prevalencia de la ideación suicida; el 30\% idealizó el suicidio. En una investigación similar desarrollada en la Universidad Federal de Mato Grosso de Brasil, se identificó al 9,9\% de estudiantes con ideación suicida. Otro estudio realizado en una universidad particular del norte de Portugal, en 366 universitarios, reveló que el 12,6\% idealizó el suicidio. Estas prevalencias se deben a los distintos instrumentos utilizados, diversas regiones y países, además del factor témporo - espacial.

Queda claro que la ideación suicida y la capacidad resiliente de afrontamiento se realizó en adolescentes de 15 y 16 años de edad; en otros enfoques investigativos esta variable cambia, como por ejemplo en estudiantes universitarios, obviamente con edades superiores. Existen variables muy complejas, que directa e indirectamente afectan a la adolescencia; esta es una etapa de cambios físicos, biológicos, neurológicos, éticos, cognitivos, etc., aspecto que es esencial en la formación integral del ser humano; es más, en la edad de 15 a 16 años, los jóvenes buscan la aceptación social y la relación de pares, como también el apoyo familiar y la independencia. En esta perspectiva, otras indagaciones 
están enfocadas en estudiantes caracterizados por una adultez temprana, esto es, han adquirido la capacidad de independencia madura y demás significativos cambios propios de la adolescencia.

Por si fuera poco, otros estudios están enfocados en la ideación suicida promovida por el consumo de sustancias, siendo este un factor crucial para que se lleve a cabo la idealización del suicidio. En este escenario multifactorial, este estudio destaca también cómo actúa la resiliencia en los adolescentes con ideación suicida, teniendo presente que hay toda una anamnesis individual matizada con las interacciones sociales que delinean la configuración del psiquismo humano.

\section{Conclusiones}

La ideación suicida es la primera etapa del pensamiento suicida. La mayoría de los adolescentes investigados exhiben un apropiado nivel de resiliencia que los habilita para enfrentar las adversidades propias de vivencias y escenarios impuros que pueden desencadenar en la ideación suicida.

Las ideas suicidas en los adolescentes están motivadas por múltiples factores, entre ellos los cambios físicos y cognitivos, la búsqueda de pares, la aceptación social, las posturas culturales, políticas, religiosas. Estos factores pueden ser vistos como irrelevantes, pero son factores predisponentes que generan las ideas autolíticas.

El 36.66\% de adolescentes investigados muestra un bajo nivel de resiliencia, de conformidad con el test SV- RES de Saavedra y Villalta; esto los convierte en personas vulnerables ante las ideas suicidas. La fortaleza emocional y cognitiva es vital para la construcción de perfiles psicológicos en los que predomine la seguridad, confianza y afectividad maduras para enfrentar los influjos nocivos que el medio socio-cultural ofrece.

La resiliencia cumple un papel tan importante como trascendental para no concretar las ideas suicidas. En los adolescentes y en todos los seres humanos representa el escudo socio-afectivo que impide el ingreso de las ideas autolíticas y de las otras fases del suicidio.

Es muy probable la identificación oportuna de los niveles de resiliencia en los adolescentes; para ello es menester la conciliación de los organismos responsables de la salud mental con el estamento educativo y familiar; así, se detectaría la ideación suicida en su fase inicial y su posterior consumación. 


\section{Bibliografía}

Arcos, A. (2016). Proyecto de investigación sobre el suicidio. Universidad de la Laguna Recuperado de: https://riull.ull.es/xmlui/bitstream/handle/915/3765/PROYECTO\%20DE\% 20INVESTIGACION\%20SOBRE\%20EL\%20SUICIDIO.pdf?sequence=1

Barrionuevo, J. (2009). Suicidios e intentos de suicidio. Recuperado de: http://suicidioprevencion. edu.uy/wp-content/uploads/2015/09/Suicidio-e-IAE-Argentina.pdf

Castañeda, K. y Piñerez, S. (2017) La prevalencia de conducta e ideación suicida con relación al género en Colombia. Universidad Cooperativa de Colombia, Bucaramanga, Colombia. Recuperado de, http://hdl.handle.net/20.500.12494/324

Cendales, R., Vanegas, C., Fierro, M., Córdoba, R., Olarte, A. (2007) Tendencias del suicidio en Colombia, 1985-2002. Rev. Panam. Salud Pública. Recuperado de: http://www.scielosp.org/pdf/rpsp/v22n4/02.pdf

De la Torre Martí, M. (2013). Protocolo para la detección y manejo inicial de la ideación suicida. Madrid: Universidad Autónoma de Madrid. Recuperado de: http://www.scielo.org.bo/scielo.php? pid $=$ S2077-21612018000100009\&script $=$ sci arttext \&tlng $=$ pt

Organización Mundial de la Salud. (2012). Prevención del suicidio (SUPRE). Recuperado de: https://www.who.int/mental health/prevention/suicide/suicideprevent/es/

Organización Mundial de la Salud. (2014). Suicidio. Recuperado de:https://www.who.int/es/newsroom/fact-sheets/detail/suicide

Pereira, A. y Cardoso, F. (2015). Suicidal Ideation in University Students: Prevalence and Association With School and Gender. Paidéia, 25(62):299-306. Recuperado de: https://dx.doi.org/10.1590/1982-43272562201503

Quinlan, M., Sanhueza, A., Espinosa, I, Escamilla J., Maddaleno, M. (2014). Suicide among young people in the Americas. J Adolesc Health, 54(3):262-8. 
Saavedra, E. , Villalta, M. (2008). Escala de Resiliencia SV-RES para jóvenes y adultos (CEANIM ed.). Recuperado de: https://www.academia.edu/26343874/Saavedra_Villalta_SVRES60 ESCALA DE RESILIENCIA SV-RES

Sánchez, J., Musitu, G., Villareal, M. y Martínez, B. (2010). Ideación Suicida en Adolescentes: Un Análisis Psicosocial. Intervención Psicosocial, 19(3), 279-287. Recuperado de: http://scielo.isciii.es/pdf/inter/v19n3/v19n3a08.pdf

Sánchez, J., Musitu, G., Villarreal, M. (2013). Adolescencia y familia. Recuperado de: https://www.uv.es/ lisis/sosa/cap13/cap12-ideacion-suic-trillas-13.pdf

Villalobos, F. (2009). Ideación suicidad en jóvenes: formulación y validación del modelo integrador explicativo en estudiantes de educación secundaria y superior. Universidad de Granada, Granada, España. Recuperado de: http://digibug.ugr.es/bitstream/handle/10481/2352/ 1805982x.pdf? sequence $=1 \&$ isAllo wed $=\mathrm{y}$

Vázquez, D., Piña, M., González, C., Jiménez, A. y Mondragón, L. (2015). La investigación sobre suicidio en México en el periodo 1980-2014: análisis y perspectivas. Acta Universitaria, 25(2). Recuperado de: http://www.actauniversitaria.ugto.mx/index.php/acta/article/ view/855/pdf 87

Varengo, J. (2016). Ideación suicida en adolescentes. Universidad siglo 21, Argentina. Recuperado de: https://repositorio.uesiglo21.edu.ar/bitstream/handle/ ues21/13032/VARENGO\%20Jud ith.pdf?sequence $=1 \&$ isAllowed $=\mathrm{y}$ 